\title{
THE NUMBER OF ISOMORPHISM TYPES OF FINITE ALGEBRAS ${ }^{1}$
}

\author{
MICHAEL A. HARRISON
}

1. Introduction. An abstract algebra is a system $\mathfrak{A}=\left\langle A, o_{i}\right\rangle_{i \in I}$ where each operation $o_{i}$ is a single valued function which assigns to every $l_{i}$-tuple of elements of $A$, say $\left(x_{1}, \cdots, x_{l_{i}}\right)$, a value $o_{i}\left(x_{1}, \cdots, x_{l_{i}}\right)$ $\in A ; l_{i}$ is the rank of $o_{i}$ for $i \in I$ and it is assumed that $l_{i} \geqq 1$ for all $i \in I$. The rank of $o$ is denoted by $\rho(o)$; the assumption $\rho(o) \geqq 1$ will be relaxed later. $\mathfrak{A}$ is said to be finite if $A$ is finite and $I$ is finite. Assume that $\mathfrak{A}=\left\langle A, o_{1}, \cdots, o_{k}\right\rangle$ is an algebra in which each operation $o_{i}$ is of rank $l_{i}$. The similarity type of $\mathfrak{A}$ is $l=\left(l_{1}, \cdots, l_{k}\right)$. Let $\mathfrak{U}=\left\langle A, o_{1}, \cdots, o_{k}\right\rangle$ and $\mathfrak{B}=\left\langle B, o_{1}^{\prime}, \cdots, o_{m}^{\prime}\right\rangle$ be two algebras; $\mathfrak{A}$ is said to be isomorphic to $\mathfrak{B}$ iff $\mathfrak{A}$ and $\mathfrak{B}$ have the same similarity type and there is a one-to-one map $\phi$ from $A$ onto $B$ such that for any $\left(x_{1}, \cdots, x_{l_{i}}\right) \in A^{l_{i}}$,

$$
\phi o_{i}\left(x_{1}, \cdots, x_{l_{i}}\right)=o_{i}\left(\phi\left(x_{1}\right), \cdots, \phi\left(x_{l_{i}}\right)\right) \quad(i=1, \cdots, k=m) .
$$

Since isomorphism is an equivalence relation, we speak of the $i s o$ morphism type of $\mathfrak{A}$ as the equivalence class containing $\mathfrak{A}$.

In this paper, the number of isomorphism types of finite algebras is derived. ${ }^{2}$ In addition, both lower bounds and algorithms for determining the number of algebras with distinguished constants are obtained. Since finite automata can be considered as finite algebras in which all the operations are unary, the present results form a generalization of the results of [4].

2. The counting method. Let $\&$ be a permutation group of order $g$ and degree $s$ acting on a set $S$. Two elements $s_{1}, s_{2} \in S$ are called equivalent iff there is an element $\alpha \in\left(S\right.$ such that $s_{1}=\alpha\left(s_{2}\right)$. The number of equivalence classes is given by a theorem due to Frobenius [1, p. 191].

TheOREM 1. Let (5) be a permutation group of order $g$ and degree $s$ acting on $S$. The number of equivalence classes induced by (5) is

$$
\frac{1}{g} \sum_{c} n_{c} I(c)
$$

Received by the editors November 9, 1964.

1 This research is sponsored by the Air Force Office of Scientific Research, Grant AF-AFOSR-639-64.

2 I wish to thank Professor A. Tarski for bringing this problem to my attention. 
where the sum is over all conjugate classes $c, n_{c}=|c|$, and $I(c)$ denotes the number of fixed points of $S$ under any permutation in $c$.

It is useful to introduce a generating function for the cycle structure of (3). Let $f_{1}, \cdots, f_{s}$ be $s$ indeterminates and let $\alpha$ be any permutation of $S$ having $j_{i}$ cycles of length $i$ for $i=1, \cdots, s$. It is convenient to use the term cycle structure of $\alpha$ and to write this $\left(j_{1}, \cdots, j_{s}\right)$ or $f_{1}^{j_{1}} \cdots f_{s}^{\jmath_{s}}$. Since every element in $S$ is in exactly one cycle of the permutation $\alpha$, we have

$$
\sum_{i=1}^{8} i j_{i}=s
$$

The cycle index polynomial of $B$ is defined to be

$$
Z_{\Theta}\left(f_{1}, \cdots, f_{s}\right)=\frac{1}{g} \sum_{(j)} g_{(j)} f_{1}^{j_{1}} \cdots f_{s}^{j_{s}},
$$

where $g_{(j)}=g_{\left(j_{1}, \cdots, j_{s}\right)}$ is the number of elements of $(5)$ having cycle structure $(j)$; the summation is over all non-negative integer solutions $(j)$ of equation (1), i.e., over all partitions of $s$.

The applications in this paper will require only the special case when $\$=\Im_{n}$, the symmetric group of degree $n$. It is well known that

$$
Z_{\Phi_{n}}\left(f_{1}, \cdots, f_{n}\right)=\frac{1}{n !} \sum_{(j)} \frac{n !}{\prod_{i=1}^{n} j_{i} ! i^{j_{i}}} f_{1}^{j_{1}} \cdots f_{n}^{j_{n}}
$$

where the summation is over all partitions of $n$.

In counting abstract algebras, it is necessary to obtain the cycle structure of a permutation induced on the cartesian product of two sets arising from permutations acting on each set individually. The following definition and theorem are due to Harary [3].

Let $\mathfrak{A}$ and $\mathfrak{B}$ be permutation groups of order $m$ and $n$ operating on disjoint object sets $X$ and $Y$ of cardinality $a$ and $b$ respectively. The cartesian product of $\mathfrak{A}$ and $\mathfrak{B}$, denoted by $\mathfrak{A} \times \mathfrak{B}$, is defined on $X \times Y$ as

$$
(\alpha, \beta)(x, y)=(\alpha(x), \beta(y))
$$

It is important to be able to compute the cycle index of $\mathfrak{A} \times \mathfrak{B}$ from the cycle indices of $\mathfrak{A}$ and of $\mathfrak{B}$. This is accomplished by defining a cross operation on cycle index polynomials. The pertinent result of Harary is the following. 
THEOREM 2 (HARARY). If

$$
Z_{\mathfrak{Q}}=\frac{1}{m} \sum_{(i)} a_{(i)} \prod_{r=1}^{a} g_{r}^{i_{r}}
$$

and

$$
Z_{\mathfrak{B}}=\frac{1}{n} \sum_{(j)} b_{(j)} \prod_{s=1}^{b} h_{s}^{j_{s}}
$$

then

$$
Z_{\mathfrak{Q} \times \mathfrak{B}}=Z_{\mathfrak{Q}} \times Z_{\mathfrak{B}}=\frac{1}{m} \frac{1}{n} \sum_{(i)} \sum_{(j)} a_{(i)} b_{(j)} \prod_{r=1}^{a} \prod_{s=1}^{b} f_{\langle r, 8>}^{i_{r} j_{\mathfrak{g}}(r, 8)},
$$

where $\langle r, s\rangle$ is the least common multiple of $r$ and $s$, while $(r, s)$ is the greatest common divisor of $r$ and $s$.

De Bruijn [2] generalized Pólya's theorem to determine the number of classes of functions under separate groups $(B)$ and $\mathfrak{S}$ acting independently on the domain and range. This result is also needed here.

Let $\$$ be a permutation group of order $g$ acting on a set $D$ of cardinality $s$ while $\mathfrak{S}$ is a permutation group of order $h$ acting on a set $R$ of cardinality $r$. Consider the class of functions from $D$ into $R$ and call two functions $f_{1}$ and $f_{2}$ equivalent if there exists an $\alpha \in B$ and a permutation $\beta \in \mathfrak{S}$ such that for every $d \in D, f_{1}(\alpha(d))=\beta f_{2}(d)$.

Theorem 3 (De BruijN). The number of classes of functions $f: D \rightarrow R$ with a permutation group (S) of a degree $s$ and order $g$ acting on $D$ and $a$ group $\mathfrak{S}$ of degree $r$ and order $h$ acting on $R$ is given by

$$
Z_{\mathfrak{S}}\left(\frac{\partial}{\partial z_{1}}, \ldots, \frac{\partial}{\partial z_{s}}\right) Z_{\mathfrak{S}}\left(h_{1}, \cdots, h_{r}\right)
$$

evaluated at $z_{1}=\cdots=z_{s}=0$, where $h_{i}=\exp \sum_{k=1}^{s / t} i z_{k i}$ for $i=1, \cdots, r$.

Theorem 3 is generally applied in actual problems through the use of the following lemma:

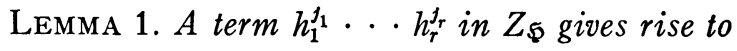

$$
Z_{\Theta}\left(\sum_{t \mid 1} t j_{t}, \cdots, \sum_{t ; s} t j_{t}\right) \text {. }
$$

The device used to generalize these classical results is to note that one may use Theorem 3 and Lemma 1 for individual terms of the 
cycle indices; the result of such computations is always the number of fixed points of the permutations. Cf. [4] for details.

3. The main results. We begin with some special cases which are of independent interest and because the final general formula is so complex.

Theorem 4. Let $\mathfrak{U}=\langle A,+\rangle$ be an algebra where $|A|=n$ and + is a binary operation on $A$, i.e. $\mathfrak{A}$ is a groupoid. The number of isomorphism types of $\mathfrak{A}$ is

$$
\frac{1}{n !} \sum_{(j)} \frac{n !}{\prod_{i=1}^{n} j_{i} ! i_{i} i_{i}} \prod_{i_{1}=1}^{n} \prod_{i_{2}=1}^{n}\left(\sum_{d\left|i_{1}, i_{2}\right\rangle} d j_{d}\right)^{j_{i_{1}} j_{i_{2}}\left(i_{1}, i_{2}\right)},
$$

where the summation is over all partitions of $n ;\left\langle i_{1}, i_{2}\right\rangle\left[\left(i_{1}, i_{2}\right)\right]$ denotes the least common multiple [greatest common divisor] of $i_{1}$ and $i_{2}$.

Proof. An isomorphism $\phi$ of $\mathfrak{A}$ onto $\mathfrak{B}$ is a one-to-one map, hence a permutation of $A$. Suppose $\phi$ has cycle structure $f_{1}^{j_{1}} \cdots f_{n}^{j_{n}}$. Since $\phi(a+b)=\phi(a)+\phi(b)$, we determine the cycle structure that $\phi$ induces on $A^{2}$. This is given by

$$
\left(f_{1}^{j_{1}} \cdots f_{n}^{j_{n}}\right) \times\left(f_{1}^{j_{1}} \cdots f_{n}^{j_{n}}\right)=\prod_{i_{1}=1}^{n} \prod_{i_{2}=1}^{n} f_{\left(i_{1}, i_{2}\right)}^{j i_{1} j i_{2}\left(i_{1}, i_{2}\right)} .
$$

Applying Lemma 1, we have that the permutation $\phi$ leaves invariant

$$
\prod_{i_{1}=1}^{n} \prod_{i_{2}=1}^{n}\left(\sum_{d \mid\left\langle i_{1}, i_{2}\right\rangle} d j_{d}\right)^{j_{i_{1}} j_{i_{2}}\left(i_{1}, i_{2}\right)}
$$

functions. Summing over all partitions of $n$ and applying Theorem 3, we have the theorem.

The next result is for abstract algebras with a single $l$-ary function.

Theorem 5. Let $\mathfrak{A}=\langle A, o\rangle$ be an algebra where $|A|=n$ and $o: A^{l}$ $\rightarrow A$. The number of isomorphism types of such algebras is

$$
\frac{1}{n} \sum_{(j)} \frac{n !}{\prod_{i=1}^{n} j_{i} ! i^{j_{i}}} \prod_{i_{1}=1}^{n} \cdots \prod_{i_{l}=1}^{n}\left(\sum_{d \backslash\left\langle i_{1}, \cdots, i_{l}\right\rangle} d j_{d}\right)^{j_{i_{1}} \cdots j_{i_{l}\left(i_{1}, \cdots, i_{l}\right)}} .
$$

Proof. Clearly

${ }^{3}$ We use $a^{\times_{k}}$ as an abbreviation for $\overbrace{a \times \cdots \times a}^{k}$ to avoid confusion with ordinary exponents. 


$$
\left(f_{1}^{j_{1}} \cdots f_{n}^{j_{n}}\right)^{\times l}=\prod_{i_{1}=1}^{n} \cdots \prod_{i_{l}=1}^{n} \underbrace{j_{i_{1}} \cdots j_{i_{l}\left(i_{1}, \cdots, i_{l}\right)}}_{\left\langle i_{1}, \cdots, i_{l}\right\rangle}
$$

The result follows from Lemma 1.

The next case is when the algebras has $k$ operations all of rank $l$.

Theorem 6. Let $\mathfrak{U}=\left\langle A, o_{1}, \cdots, o_{k}\right\rangle$ be an algebra where $|A|=n$ and $o_{i}: A^{l} \rightarrow A$ for each $i=1, \cdots, k$. The number of isomorphism types is

$$
\frac{1}{n !} \sum_{(j)} \frac{n !}{\prod_{i=1}^{n} j_{i} ! i^{j_{i}}} \prod_{i_{1}=1}^{n} \cdots \prod_{i_{l}=1}^{n}\left(\sum_{d \mid\left\langle i_{1}, \cdots, i_{l}\right\rangle} d j_{d}\right)^{k j_{i_{1}} \cdots j_{i l}\left(i_{1}, \cdots, i_{l}\right)} .
$$

Proof. The result follows from the previous theorem upon noting that each of the $k$ operations must be considered independently.

Lastly, we consider the general case of $k$ operations where each $\boldsymbol{o}_{\boldsymbol{i}}$ has rank $l_{i}$. No loss of generality results from permuting the operations so as to keep operations of the same rank adjacent.

Theorem 7. Let $\mathfrak{A}=\left\langle A, o_{1}, \cdots, o_{k}\right\rangle$ where each $o_{i}: A^{l_{i} \rightarrow A}$ and $|A|=n$; assume that there are $m_{r}$ operations of rank $r$ for $r=1, \cdots$, $\max _{i}\left(\rho\left(o_{i}\right)\right)=q$. The number of isomorphism types of such algebras is

$$
\frac{1}{n !} \sum_{(j)} \frac{n !}{\prod_{i=1}^{n} j_{i} ! i_{i_{i}}} \prod_{r=1}^{q}\left(\prod_{i_{1}=1}^{n} \cdots \prod_{i_{l r}=1}^{n} \sum_{d \backslash\left\langle i_{1}, \cdots, i_{l r}\right\rangle} d j_{d}\right)^{m_{l_{r}} j_{i_{1}} \cdots j_{i_{l}}\left(i_{1}, \cdots i_{l_{r}}\right)} .
$$

4. Further results. It is often convenient to consider algebras with distinguished constants, e.g., $\langle R,+, \cdot, o, 1\rangle$. We identify constants with functions of rank 0 . The number of isomorphism types of such algebras is obtained by using the same trick employed in [4] to count automata with fixed initial states. ${ }^{4}$

Theorem 8. Let $\mathfrak{A}=\left\langle A, o_{1}, \cdots, o_{k}, c_{1}, \cdots, c_{m}\right\rangle$ be an abstract algebra with $|A|=n \geqq m$. Let $F_{\mathfrak{Q}^{\prime}}(n)$ be the formula for the number of isomorphism types of algebras $\mathfrak{H}^{\prime}=\left\langle A, o_{1}, \cdots, o_{k}\right\rangle$ with $|A|=n$ as given by Theorem 7. The number of isomorphism types of algebras $\mathfrak{U}=\left\langle A, o_{1}, \cdots, o_{k}, c_{1}, \cdots, c_{m}\right\rangle$ is given by

$$
S_{j_{1}+m}^{j_{1}} F_{\mathfrak{I}^{\prime}}(n-m)
$$

where the formula means that $j_{1}$ is replaced by $j_{1}+m$ throughout $F_{\mathfrak{Q}}(n-m)$.

- I believe that this device is originally due to Frank Harary who used it to count nonisomorphic rooted graphs. 
TABLE 1. The number of types of algebras with one binary operation.

\begin{tabular}{c|c}
$n$ & Number of Types \\
\hline 1 & 1 \\
\hline 2 & 10 \\
\hline 3 & 3,330 \\
\hline 4 & $178,981,952$ \\
\hline
\end{tabular}

TABLE 2. The number of types of algebras with two binary operations.

\begin{tabular}{c|c}
$n$ & Number of Types \\
\hline 1 & 1 \\
\hline 2 & 136 \\
\hline 3 & $64,573,605$ \\
\hline
\end{tabular}

Proof. Under any isomorphism $\phi$ of $\mathfrak{A}=\left\langle A, o_{i}, \cdots, o_{k}, c_{1}, \cdots, c_{m}\right\rangle$ on to $\mathfrak{B}=\left\langle B, o_{1}{ }^{\prime}, \cdots, o_{1}{ }^{\prime}, c_{1}{ }^{\prime} \cdots, c_{m}{ }^{\prime}\right\rangle$ we must have $\phi\left(c_{i}\right)=c_{i}^{\prime}$ for $i=1, \cdots, m$. The $(n-m)$ remaining elements may be permuted by $\phi$ as before while the constants remain fixed.

Since the formulas of $\S 3$ are complex, it would be desirable to have simple estimates for the desired numbers. One immediately obtains the following lower bound in the general case,

$$
\frac{1}{n !} \prod_{r=1}^{q} n^{m} l_{r} n^{l r}
$$

by just taking the first term of the formula. In [4] and hence for unary algebras, the lower bound has also been shown to be the asymptotic estimate as $n$ increases without limit. This simple result does not generalize to the present case. It would be desirable to obtain asymptotic estimates but this task falls outside the context of the present paper.

5. Numerical results. We present some numerical results in the most interesting cases. Since the number of types grows so rapidly, only very small values of $n$ are considered. Note that the results of Table 4 as compared with Table 3 confirm that, with designated constants, the number of isomorphism types increases since there is less freedom in constructing isomorphisms. 
TABLE 3. The number of types of algebras with one ternary operation.

\begin{tabular}{l|l}
$n$ & Number of Types \\
\hline 1 & 1 \\
\hline 2 & 130 \\
\hline 3 & $1,270,932,917,454$ \\
\hline
\end{tabular}

TABLE 4. The number of types of algebras with one binary operation and one constant.

\begin{tabular}{c|c}
$n$ & Number of Types \\
\hline 1 & 1 \\
\hline 2 & 16 \\
\hline 3 & 9,882 \\
\hline
\end{tabular}

6. Unsolved problems. While the present paper presents a complete solution to the problem of enumerating finite algebras, our methods do not directly extend to the problems of enumerating the types of finite algebraic systems, such as monoids, groups and rings. The present approach must be modified to take into account the additional axioms which the operations must satisfy. Clearly, associativity should be studied first.

\section{REFERENCES}

1. W. Burnside, Theory of groups of finite order, Cambridge Univ. Press, New York, 1911.

2. N. G. De Bruijn, Generalization of Polya's fundamental theorem in enumerative combinatorial analysis, Nederl. Akad. Wetensch. Proc. Ser. A 52 (1959), 59-69.

3. F. Harary, On the number of bi-colored graphs, Pacific J. Math. 8 (1958), 743-755.

4. M. A. Harrison, A census of finite automata, Canad. J. Math. 17 (1965), 100-113.

University of California, Berkeley 